\title{
Real Time Closed Loop Control of Full Penetration Keyhole Welding with Cellular Neural Network Cameras
}

\author{
F. Abt ${ }^{* 1}$, A. Blug ${ }^{2}$, L. Nicolosi ${ }^{3}$, F. Dausinger ${ }^{4}$, H. Höfler ${ }^{2}$, R. Tetzlaff ${ }^{3}$, R. Weber ${ }^{5}$ \\ ${ }^{* 1}$ FGSW Forschungsgesellschaft für Strahlwerkzeuge gGmbH, Paffenwaldring 43, 70569 Stuttgart, Germany, \\ E-mail:felix.abt@fgsw.de \\ ${ }^{* 2}$ Fraunhofer Institute for Physical Measurement Techniques IPM, Heidenhofstr. 8, 79110 Freiburg, Germany \\ ${ }^{*}$ IEE Institut fuer Grundlagen der Elektrotechnik u. Elektronik, Mommsenstr. 12, 01069 Dresden, Germany \\ ${ }^{* 4}$ Dausinger \& Giesen GmbH, Rotebühlstr. 87, 70178 Stuttgart, Germany \\ *5 IFSW Institut fuer Strahlwerkzeuge, Paffenwaldring 43, 70569 Stuttgart, Germany
}

\begin{abstract}
Camera based in-process control for laser welding enables flexible image processing which allows the adaption of the system to different processes and quality features. A closed loop control system with a Cellular Neural Network Camera was implemented into a laser welding machine. The system is surveying the contour of the full penetration hole with a frame rate of over $10 \mathrm{kHz}$ for both, acquisition and evaluation of area images. As a result the system reaches and holds the full penetration state automatically. This paper shows the latest experimental results including the extension to direction independent weldings.
\end{abstract}

DOI:10.2961/jlmn.2011.02.0007

Keywords: cellular neural network (CNN), process control, closed loop, laser welding

\section{Introduction}

Error prevention and correction is a key to fast and reliable laser welding processes from automobile production to micro mechanics. Closed loop control systems can help to achieve high productivity and constant quality even under changing welding conditions. Camera based in-process control allows to employ algorithms for image processing which are widely independent of the exact process parameters, reducing the adaption effort for different applications compared to simple photo diode systems.

Unfortunately, most of today's conventional micro processor based image processing architectures [1] work at frame rates which are not sufficient for real-time camera based closed loop control of high speed laser welding.

With "Cellular Neural Networks" (CNN) it is possible to integrate processor elements in the electronic circuitry of CMOS cameras resulting in a Single-Instruction-MultipleData (SIMD)-architecture on the camera chip itself. Such pixel parallel systems provide extremely fast real-time image processing.

This article shows the potential of a closed loop control system based on CNN-technology. The system is surveying the contour of the full penetration hole with a control frequency in the multi kilohertz range. As a result the system reaches and holds the full penetration state automatically.

\section{CNN-Technology}

CNN have been introduced by Chua and Yang in 1988 [2] to be employed in such areas like image processing and pattern recognition. The basic circuit unit of CNNs is called a cell. Each cell can directly interact with its neighbouring cells since it is connected to them, and indirectly with the other cells due to propagation effects caused by the continuous-time dynamics of the CNN.

An example of a two-dimensional $\mathrm{CNN}$ is shown in Fig. 1.

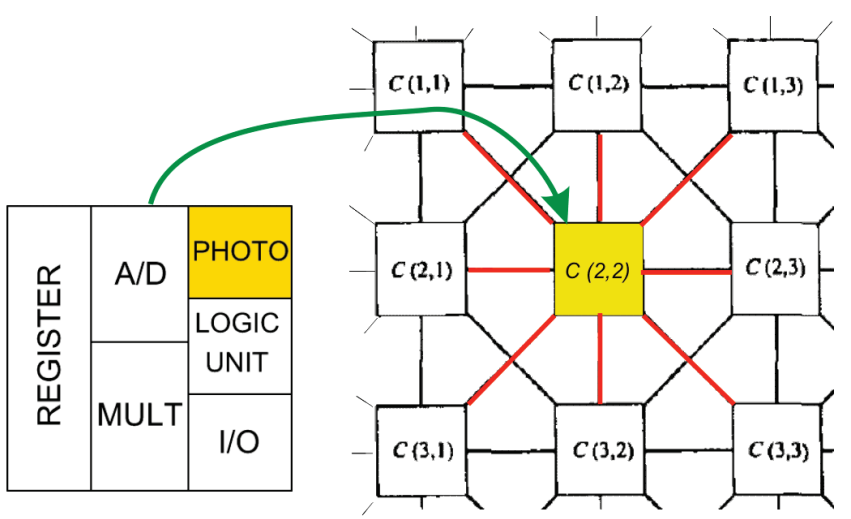

Fig. $12 \mathrm{D} \mathrm{CNN}$ scheme: each cell is directly connected with its neighbouring cells. It can interact with all other cells due to propagation effects. Each of the cells consists of a photo sensitive area and a surrounding analogue processor unit.

The CNN analogue implementation allows to perform parallel processing since each pixel has its own processor element and the cell interaction occurs in a really short time determined by the time constant of the single cell. This makes the CNN one of the best approaches for real-time image processing. A more detailed description of the $\mathrm{CNN}$ technology is given in [2], [3], [4] and [5]. 


\section{The Laser Welding Process}

The relevant process for this paper is the keyhole welding of zinc-coated steel in an overlap-joint with solid state lasers. Fig. 2 shows the sketch of a longitudinal section of the laser welding process and the resulting image of a coaxial camera in the near infrared spectrum. The camera image shows the thermal radiation of the process in the range between $820 \mathrm{~nm}$ and $980 \mathrm{~nm}$.

As one can see in Fig. 2, the full-penetration quality feature is directly visible in the camera image as a dark area following the interaction zone of the laser. This is because the capillary is fully open at the bottom of the joining partners and thus there is no molten material that emits thermal radiation in this area. This was also observed in previous publications [6], [7].

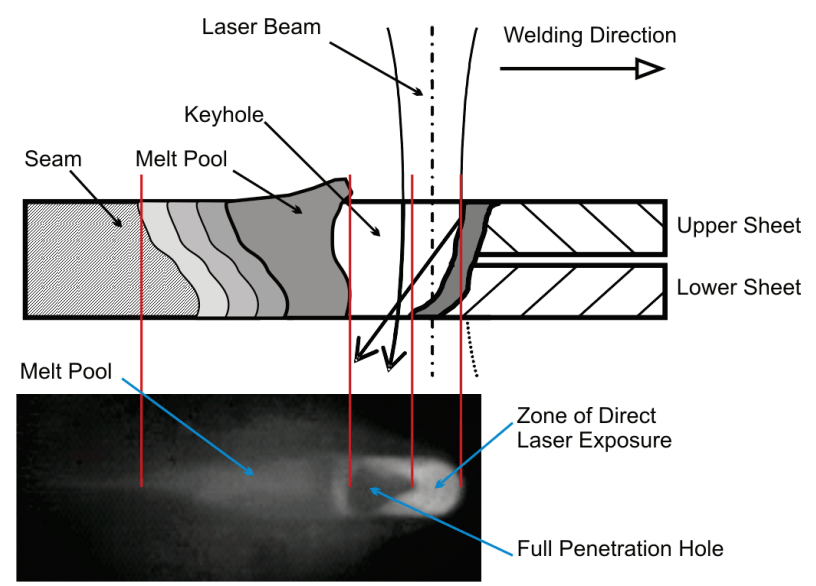

Fig. 2 Schematics of a welding process in an overlap-joint.

\section{Experimental Setup}

The welding experiments were carried out with a 2D laser scanner setup. The laser source for the experiments is a $6 \mathrm{~kW}, 1030 \mathrm{~nm}$ Trumpf TruDisk $6002 \mathrm{Yb}$ :YAG thin disk with a $200 \mu \mathrm{m}$ transport fibre. The laser scanner used - a Trumpf PFO-33 - was equipped with a $450 \mathrm{~mm}$ focusing optic which resulted in a focal diameter of $600 \mu \mathrm{m}$.

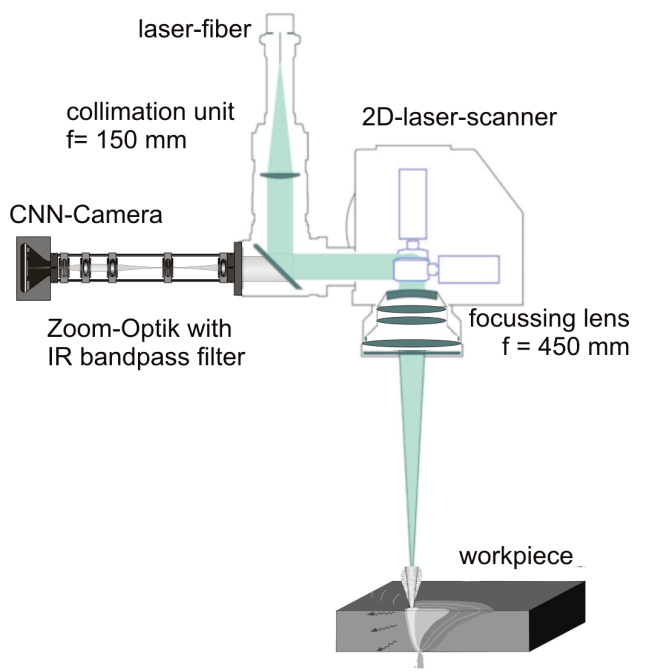

Fig.3 Optical setup with the laser scanner, the $\mathrm{CNN}$-camera and the optical imaging system.
The Eye-RIS CNN-camera system is adapted to the scanner optic through a $90^{\circ}$ beam splitter, see Fig. 3. Thus the camera perspective is coaxial to the laser beam. This allows an invariant field of view regardless of the scanner position. It is necessary to use an aligned lens system in order to achieve an appropriated region of interest (ROI) for the camera. For the experiments a lens system consisting of three achromatic lenses was designed to achieve an optical magnification $\beta$ of about 4.6. In combination with an optical band-pass filter - transmission from $820 \mathrm{~nm}$ up to $980 \mathrm{~nm}$ - camera images with high quality can be achieved [3].

\section{Hardware and Algorithms}

\subsection{Camera Hardware}

In the recent years, Cellular Neural Networks (CNN) based technologies were used to integrate massively parallel "Single Instruction Multiple Data" (SIMD) processor architectures in camera chips [14][15]. Here, a system called Q-Eye developed by Anafocus [13] was used to execute the algorithms for the detection of the full penetration hole. The Q-Eye is a quarter CIF resolution fully programmable smart image sensor. It consists of 176 x 144 cells, each containing a processor merged with an optical sensor, memory circuitry and interconnections to the 8 neighbouring cells. This means that each pixel can both sense the corresponding spatial sample of the image and process this information in close interaction with other pixels.

The Q-Eye is part of the Eye-RIS camera which contains an additional FPGA based NIOS II processor by Altera to control the operation of the whole vision system and to analyze the information output of the SIS performing all the decision-making and actuation tasks.

\subsection{Algorithm}

As shown in earlier works [8] the intensity image of the laser interaction zone is rather constant for a large range of laser power and feeding rates. Therefore, a global threshold is applicable to binarize the images acquired during the welding process. An example of binarized image is shown in Fig. 4 (a). In the following algorithms to discriminate the full penetration hole by the execution of directional dilations are described [9]. Since they are based on basic local neighborhood operations [10], they can be implemented and efficiently run on any $\mathrm{CNN}$ architecture. Fig. 4 shows the result obtained executing dilation from the top - right corner to the bottom - left corner of the binarized image [11].

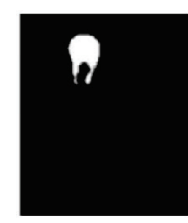

(a)

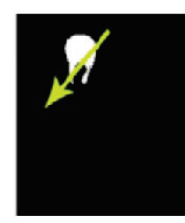

(b)

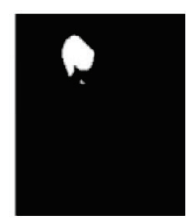

(c)

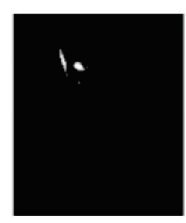

(d)
Fig. 4 Dilation parallel to one diagonal. (a) is the source image; (b) shows the direction of the dilations; (c) is the result of the dilating operation. At the end, a logical XOR and a logical AND are applied in succession in order to keep white pixels in the dilated area only (d) [11]. 
The algorithm applies a constant threshold value to the source image and performs a dilating operations along the image diagonal. Afterwards, logical operations are executed in order to keep white pixels in the dilated area only, as described in Fig. 4. At the end, the mask is applied in order to hide the noise due to the dilation of the external interaction zone edges. The algorithm and the application of the masking operation take a single image processing time of about $42 \mu$ s. Fig. 5 clarifies the principle of the algorithm [11].

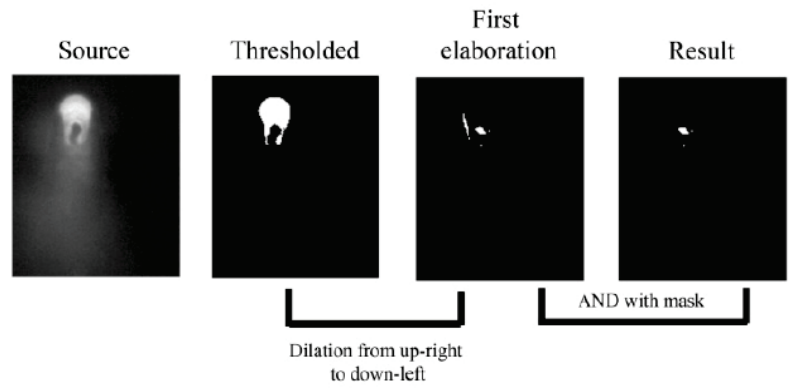

Fig. 5 Image evaluation algorithm. The source image is at first binarized and then dilated along the image diagonal from the topright to the bottom-left. The dilating result is masked at the end [11].

\subsection{Maskbuilder}

The mask builder is a tool which automatically creates one mask at the beginning of the welding process in order to make the mask independent of the specific optical set-up. The idea is to estimate the position of the interaction zone centre in order to align the mask with the key hole. Let's consider the example in Fig. 6.

The first step consists in creating an initial mask, which is vertically divided in two regions (black and white) of the same size, as in picture (d). Afterwards, the source image (a) is binarized obtaining the picture (b) and the distance of the interaction zone centre from the image centre is estimated, as in (c). By this information, the initial mask is opportunely shifted in order to be overlapped to the interaction zone centre, as in picture (e). An example of masking operation is showed in (f). A more detailed description of the mask builder algorithm is given in [11].

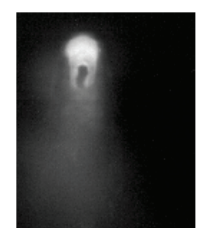

(a)

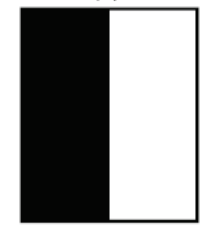

(d)

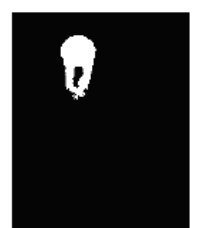

(b)

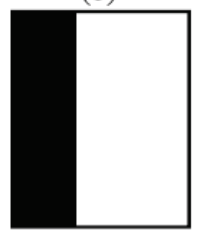

(e)

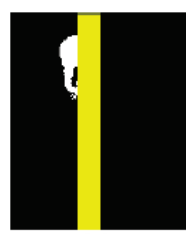

(c)

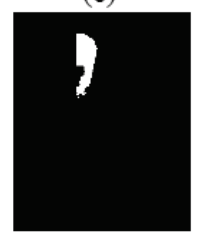

(f)
Fig. 6 Mask builder. The source image (a) is binarized (b) and the distance of the interaction zone from the image centre is estimated, as in (c). Afterwards, the initial mask (d) is shifted to be overlapped with the centre of the interaction zone, as in (e). Picture (f) shows the application of the masking operation over the source image [11].

\subsection{Control System}

As mentioned above, this paper deals with an algorithm implementation for closed loop tests; i.e. the CNN-camera system controls the laser welding machine. The available hardware therefore essentially consists of: the Q-Eye, a numerically controlled (NC) machine, the laser machine and the interface board. The NC machine is responsible for safety interlocks, laser ON/OFF signals and movement. The laser power is controlled by an analog voltage between 0 and $10 \mathrm{~V}$ which corresponds to $0-100 \%$ of the laser power. The interface board was built in order to receive a digital START/STOP signal from the NC machine and to control the laser power according to the pulse width modulation (PWM) signal received from the $\mathrm{CNN}$-camera, see Fig. 7.

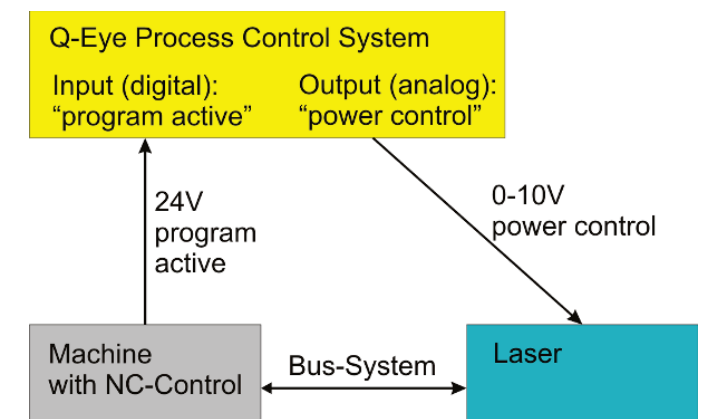

Fig. 7 Logical connections between the Q-Eye, the NC-control and the laser.

The exposure time of the camera is approximately 40 $\mu \mathrm{s}$. By calculating the total processing time of a controlled laser welding machine, the sensing function execution time of $15-20 \mu$ s and the communication function execution time of around $20 \mu \mathrm{s}$ have to be taken into account. The communication functions are used to receive the digital signal from the machine control system and to send the right PWM signal to the machine, in order to set the laser power according to the result of the image evaluation. The total time consumption for one image is about $72 \mu \mathrm{s}$. This leads to a control frequency between 10 and $15 \mathrm{kHz}$.

\section{Closed loop control for full-penetration in a real welding process}

As described above, the real-time control test employed the full-penetration quality feature. Therefore, fullpenetration is detected by the presence or absence of the dark area behind the laser interaction zone, as described in previous chapters. The actuating variable used was the laser power. The advantages of this actuating variable are the high dynamic of up to $10 \mathrm{kHz}$ for Trumpf laser systems and the fact that it can be controlled by a simple analogue signal of $0-10 \mathrm{~V}$ corresponding to a laser power output of 0 $-100 \%$

As further described in the chapters above, we were able to reach a control frequency of about $13.8 \mathrm{kHz}$. This was achieved without saving the camera images, which would have dropped the control frequency to approximately $3 \mathrm{kHz}$. The resulting benefits are described in the following subchapters [3]. 


\subsection{Comparison controlled vs. uncontrolled}

The conventional way to achieve a proper fullpenetration weld is to regulate laser power or feeding rate until full-penetration is reached and add $10 \%$ laser power as a safety factor.

Such a conventional uncontrolled full-penetration weld is shown in Fig. 8. Typical for this process is the significant contamination of the bottom side of the joining partners with smoke residue and spatters. Furthermore, there are usually significant craters or even holes present especially at the end of the weld seam due to the deceleration of the machine axis at the end of the welding process.

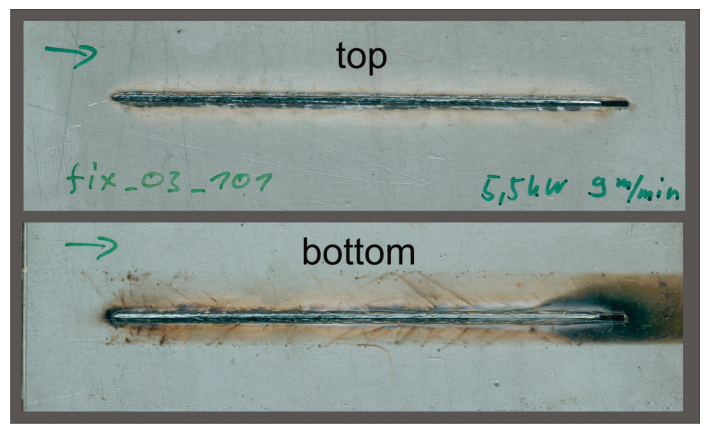

Fig. 8 Uncontrolled full-penetration weld with $10 \%$ factor of safety. Parameters are $\mathrm{v}=9 \mathrm{~m} / \mathrm{min}, \mathrm{P}=5.5 \mathrm{~kW}$, zinc coated steel $2 \times 0.7 \mathrm{~mm}$ with $0.1 \mathrm{~mm}$ gap in an overlap joint [3].

With the controlled full-penetration welding process one can see a completely different behaviour (Fig. 9). Notable is the almost complete absence of smoke residue on the bottom side of the joining partners and the reduced spatter traces.

It is also visible that the Eye-RIS control system reduces the laser power automatically at the end of the weld seam when the feeding rate decreases to a halt. The laser power does not drop below under $2 \mathrm{~kW}$ because this is the minimum power preset for that experiment. This reduces the formation of craters and holes at weld termination [3].

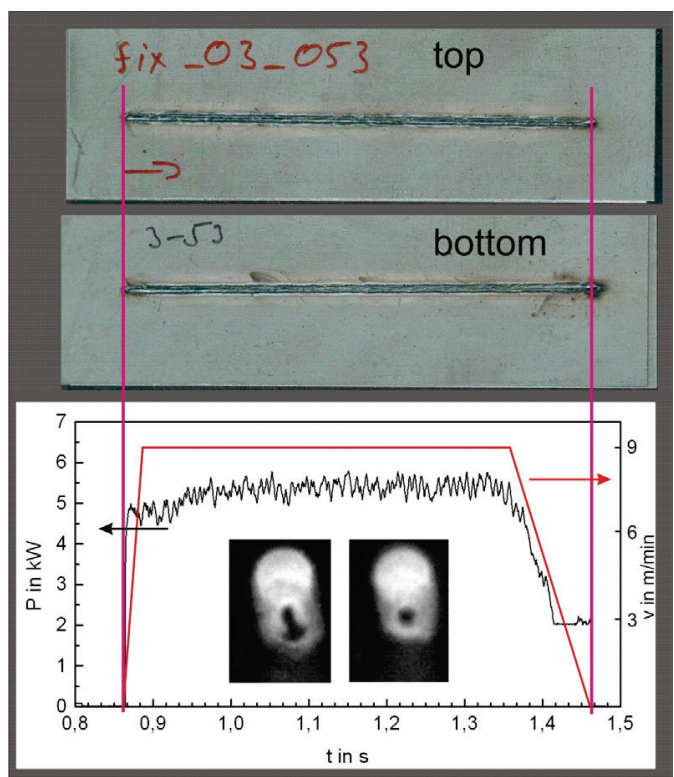

Fig. 9 Controlled full-penetration weld of zinc coated steel $2 \mathrm{x}$ $0.7 \mathrm{~mm}$ with $0.1 \mathrm{~mm}$ gap at a feeding rate of $\mathrm{v}=9 \mathrm{~m} / \mathrm{min}$ including two exemplarily camera pictures of a good full-penetration state [3].

\subsection{Laser Power Adaption to Feeding Rate}

The adaption of the laser power to feeding rate was tested by welding the same material at changing feeding rates between $3 \mathrm{~m} / \mathrm{min}$ and $9 \mathrm{~m} / \mathrm{min}$. As Fig. 10 illustrates, the laser power was adjusted properly by the control system to the particular feeding rate.

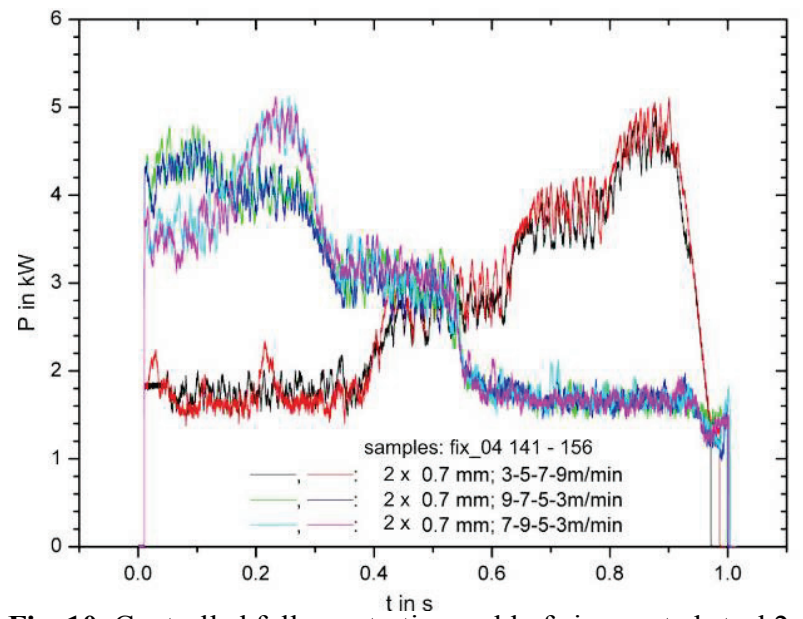

Fig. 10 Controlled full-penetration weld of zinc coated steel $2 \times$ $0.7 \mathrm{~mm}$ with $0.1 \mathrm{~mm}$ gap at different feeding rates between 3 $\mathrm{m} / \mathrm{min}$ and $9 \mathrm{~m} / \mathrm{min}$.

Fig. 11 shows the corresponding weld seams of speed variation in Fig. 10. The full-penetration state was reached and maintained in all cases.

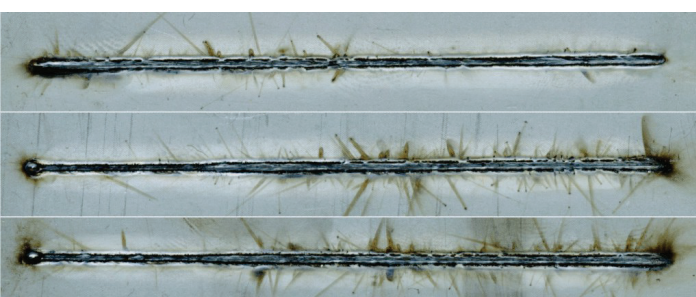

Fig. 11 Corresponding weld seams (bottom side) of the feeding rate variation. Top: fix 04141 with 3-5-7-9 $\mathrm{m} / \mathrm{min}$, middle: fix_04_143 with 9-7-5-3 $\overline{\mathrm{m}} / \mathrm{min}$, bottom: fix_04_145 with 7-9-5-3 $\mathrm{m} / \mathrm{min}$. The welding speed changes every $20 \mathrm{~mm}$.

\subsection{Laser Power Adaption to Material Thickness}

Experiments with changing thickness of the top sheet where carried out to test the ability of the control system to maintain the full-penetration at variable material thicknesses of the joining partners.

Fig. 12 shows the positive step response of the control system to a change in the material thickness. The position of the thickness changing is marked in the diagram to measure the necessary time to change the laser power. With the control time and the power difference, it is possible to calculate the effective control speed of the system. For the experiments in Fig. 12, an effective control speed between $23 \mathrm{~kW} / \mathrm{s}$ and $39 \mathrm{~kW} / \mathrm{s}$ was achieved. The effective control speed refers to the achievement of average power levels, not to the maximum power gradient, that is approximately $165 \mathrm{~kW} / \mathrm{s}$.

Exemplarily Fig. 13 shows the weld seams of two of the experiments out of Fig. 12. The full-penetration was reached and maintained across the thickness step in the middle of the seam. The quality of the top and bottom side is good, without holes and penetration losses. 


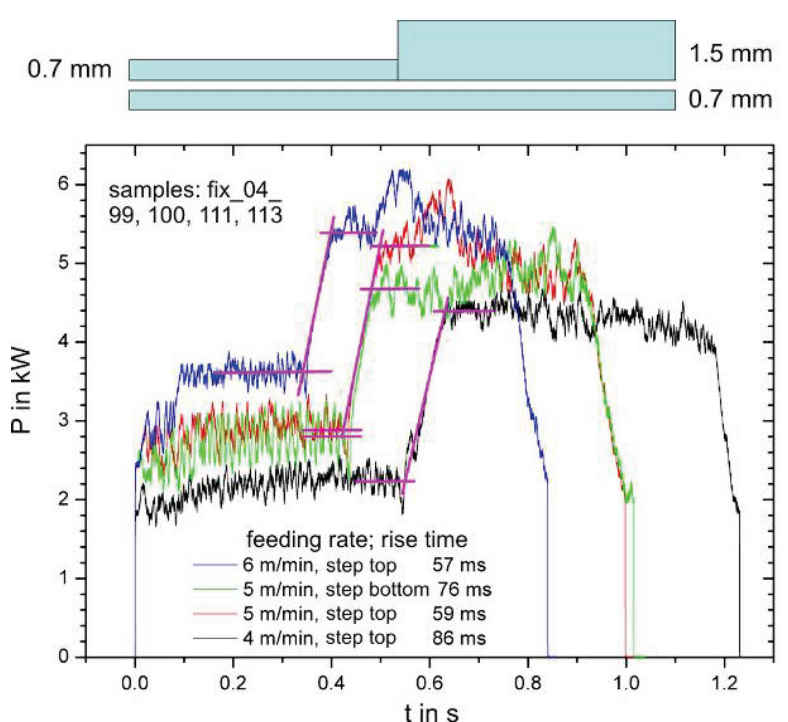

Fig. 12 Controlled full-penetration weld of zinc coated steel. The material thickness changes form $2 \times 0.7 \mathrm{~mm}$ to $0.7 \mathrm{~mm}+1.5 \mathrm{~mm}$ in the middle of the weld seam. The gap is $0.1 \mathrm{~mm}$ in all cases.

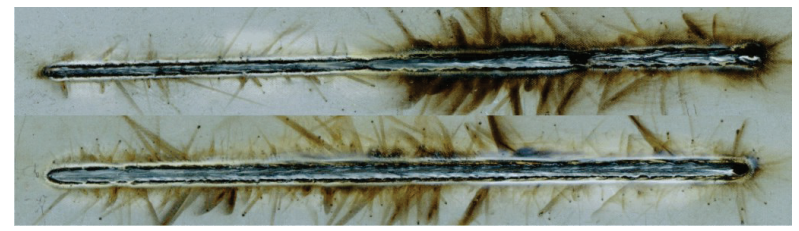

Fig. 13 Bottom sides of weld seams with $0.7 \mathrm{~mm}$ bottom sheet and thickness change from $0.7 \mathrm{~mm}$ to $1.5 \mathrm{~mm}$ in the top sheet in the middle of the seam. Top: fix 04099 at $5 \mathrm{~m} / \mathrm{min}$, bottom:

$$
\text { fix_04_113 at } 6 \mathrm{~m} / \mathrm{min} \text {. }
$$

\subsection{Robustness against changing gap size}

To test the ability of the control system to deal with variable gap sizes between the sheets, some tests were done with opening gaps from $0.0 \mathrm{~mm}$ to $0.6 \mathrm{~mm}$ at material thicknesses of $2 \times 0.7 \mathrm{~mm}$.

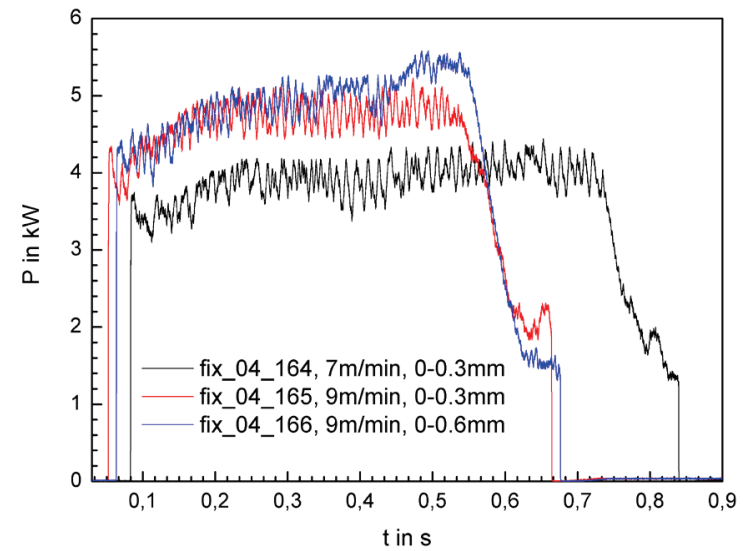

Fig. 14 Opening gap at $2 \times 0.7 \mathrm{~mm}$ material and different feed rates. [16].

Fig. 14 shows the graph of the controlled laser power for opening gaps. The gap has virtually no influence on the controlled laser power and the full penetration was ensured over the whole weld seam. Sample fix_04_166 suffered a "false friend" from the middle of the process. But the difference in the behaviour of the laser power was not very significant, which makes it hard to detect the "false friend".

\section{Direction Independent Algorithm}

A new algorithm for direction independent welding processes is currently in testing and optimization.

The so called Omnidirectional algorithm for welding processes is also based on local neighborhood operations and, therefore, it is expected to run efficiently on any $\mathrm{CNN}$ architecture.

The first step consists in enhancing the source image contrast by the application of sharpening filters. Afterwards, the resulting image is binarized with a global threshold like in the algorithm described in chapter 5.2. The second step consists in detecting the full penetration hole. At first, iterative morphological closings are executed, in order to fill the full penetration hole. The application of a logical XOR and a logical AND comes after, in order to keep white pixels in the hole area only. The last step is the noise reduction by the use of morphological openings and by the subsequent application of a mask.

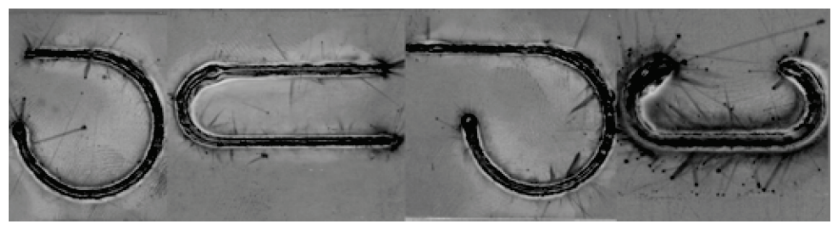

Fig. 15 Some welding examples with variable welding direction

[12].

Fig. 15 shows some welding experiments with variable orientation. Simulations showed that Omnidirectional algorithm and masking operation lead to a single image processing time of about $110 \mu \mathrm{s}$. Hit rates of the image elaboration between $85 \%$ and $98 \%$ were reached in the simulation. More details can be found in [12].

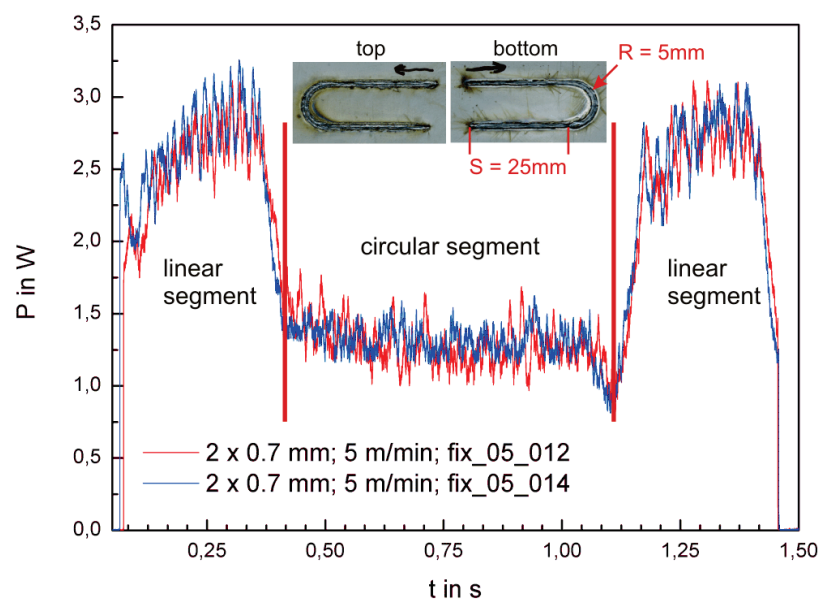

Fig. 16 Controlled full-penetration weld of zinc coated steel. The material thickness changes form $2 \times 0.7 \mathrm{~mm}$ with a gap of $0.1 \mathrm{~mm}$ in both cases. The weld seam starts with a $25 \mathrm{~mm}$ linear segment followed by a $180^{\circ}$ circle with a radius of $5 \mathrm{~mm}$ and ends after another linear segment of $25 \mathrm{~mm}$ length.

The Omnidirectional algorithm offers good stability and repeatability, as illustrated in Fig. 16. The federate of the whole weld seam was set to $5 \mathrm{~m} / \mathrm{min}$. Due to the limited dynamics of the mechanical axis of the xy-stage, the speed dropped to approximately $2 \mathrm{~m} / \mathrm{min}$ in the circular section. The control system was able to follow the machine dynamics to maintain a suitable power level to achieve a sound weld. 
A comparable test was performed to have speed steps with different heights. Fig. 17 shows results of the test where the linear segment was welded with different speeds between $3 \mathrm{~m} / \mathrm{min}$ and $7 \mathrm{~m} / \mathrm{min}$. In the successive circular section the speed dropped to approximately $2 \mathrm{~m} / \mathrm{min}$. The control system was abele to reach the full penetration in the linear segment automatically and to reduce the power to a suitable value for the circular section. The repeatability was very high as can be seen in Fig. 17 where the power level in the circular section is almost the same for all experiments.

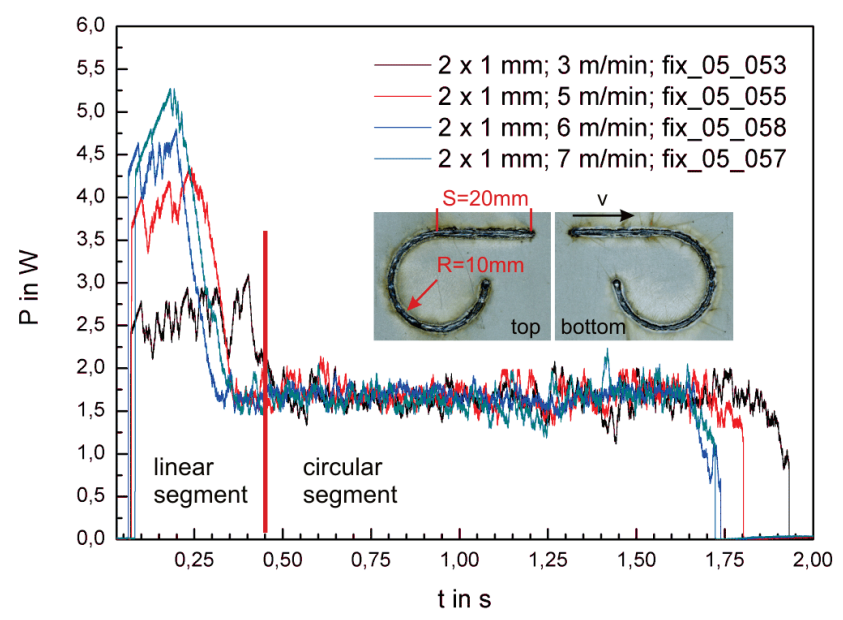

Fig. 17 Controlled full-penetration weld of zinc coated steel. The material thickness changes form $2 \times 1 \mathrm{~mm}$ with a gap of $0.2 \mathrm{~mm}$ in all cases. The weld seam starts with a $20 \mathrm{~mm}$ linear segment followed by a $270^{\circ}$ circle with a radius of $10 \mathrm{~mm}$.

\section{Conclusion}

The application of algorithms which exploit local neighbourhoods on $\mathrm{CNN}$-architectures allows for a significant enhancement to the frame rate for camera based process control systems in laser welding. As for the fullpenetration quality feature, it was shown that a real-time control system, based on image processing with a CNNcamera, enables frame rates of up to $13.8 \mathrm{kHz}$ for linear welding processes and up to $9 \mathrm{kHz}$ for direction independent processes.

The major benefit for the laser welding process is a robust and fast contour detection of the rapidly changing full penetration hole. Based on this contour detection, an instant feedback signal for the laser power is generated. In that sense, the system marks the step from image based process monitoring to closed loop control systems which maintain the desired state of the process under a larger range of parameters. As demonstrated, the control system can handle different feeding rates as well as variable thickness of the joining partners.

The variation in thickness was limited in this study in the range of $0.7 \mathrm{~mm}$ up to $1.5 \mathrm{~mm}$. In other publications like [16] and [17] experiments with material thicknesses of up to $2 \times 2.5 \mathrm{~mm}$ were carried out successfully with this control system.

The quality of the controlled weld seam is better than the uncontrolled one. There is a significant reduction in smoke residue and spatters on the bottom side of the mate- rial. In addition, the laser power is automatically reduced at lower feeding rates at the end of the welding process thus effectively preventing the formation of craters and holes at the end of the weld seam.

The CNN-based control system has proved its ability to meet the requirements for real-time high speed camera based process control in laser welding. In further experiments and projects, the system will be improved to cover a wider variety of quality features and other processes.

\section{References}

[1] J. Müller-Borhanian, J. Hohenadel, M. KogelHollacher, D. Pfitzner, C. Deininger, A. Kattwinkel, T. Wiesendanger, A. Ruprecht; Integration optischer Messmethoden zur Prozesskontrolle beim Laserstrahlschweißen, 2005, Herbert Utz Verlag, München, Germany.

[2] Leon O. Chua, Fellow, IEEE, and Lin Yang, student member, IEEE, "Cellular Neural Networks: Theory", IEEE TRANSACTIONS ON CIRCUITS AND SYSTEMS, VOL. 35, NO. 10, OCT. 1988.

[3] Abt, F.; Nicolosi, L.;Carl, D.; Blug, A.; Geese, M.; Dausinger, F.; Deininger, C.; Höfler, H.; Tetzlaff, R.: CLOSED LOOP CONTROL OF LASER WELDING PROCESSES WITH CELLULAR NEURAL NETWORK (CNN) CAMERAS, Proc. of the 27th International Congress on Applications of Lasers \& Electro-Optics (ICALEO 2008), 2008, Temecula, CA, USA, 817-825.

[4] Blug, A.; Carl, D.; Höfler, H.; Abt, F.; Geese, M.; Tetzlaff, R.: Pixelparallele Bildverarbeitung mit CNN zur Regelung von Laserschweiß-prozessen, VDIBerichte, Sensoren und Messsysteme 2008, Vol. 2011, 2008.

[5] F. Corinto, M. Gilli and P.P. Civalleri, Dipartimento di Elettronica, Politecnico di Torino, Corso Duca degli Abruzzi 24, I-10129 Torino, Italy, On stability of full range and polynomial type CNNs, in Proc. of the 7th IEEE International Workshop on Cellular Neural Networks and their Applications, pp.33-40.

[6] Fabbro, R.; Coste, F.; Goebels, D.; Kielwasser, M. (2004) Study of Nd-YAG Welding of Zn-Coated Steel Sheets, in Proceedings of the 23nd International Congress on Applications of Lasers and Electro-Optics, ICALEO 2004, San Francisco, USA.

[7] Bardin, F.; Cobo, A.; Lopez-Higuera, J.; Collin, O.; Aubry, P.; Dubois, T.; Högström, M.; Nylen, P.; Jonsson, P.; Jones, J.; Hand, D. (2004) Process Control of Laser Keyhole Welding, in Proceedings of the 23nd International Congress on Applications of Lasers and Electro-Optics, ICALEO 2004, San Francisco, USA.

[8] M. Geese, R. Tetzlaff, D. Carl, A. Blug, H. Höfler, F. Abt, (2008) Feature Extraction in Laser Welding Processes, in Proc. of the 11th International Workshop on Cellular Neural Networks and their Applications CNNA 2008, Santiago de Compostela, Spain.

[9] L. Nicolosi, R. Tetzlaff, F. Abt, A. Blug, H. Höfler, D. Carl, New CNN based algorithms for the full penetration hole extraction in laser welding processes, IEEE International Symposium on Circuits and Systems ISCAS, May 24-27 2009, Taipei, Taiwan (to be pub- 
lished).

[10]L.O. Chua, T. Roska, Cellular neural networks and visual computing. Foundations and applications, Cambridge University Press 2004, first published in printed format 2002.

[11] Nicolosi, L.; Tetzlaff, R.; Abt, F.; Blug, A.; Carl, D.; Höfler, H.: New CNN Based Algorithms for the Full Penetration Hole Extraction in Laser Welding Processes: Experimental Results, in Proceedings of the International Joint Conference on Neural Networks IJCNN 2009, Atlanta, GA, USA (to be published).

[12]Nicolosi, L.; Tetzlaff, R.; Höfler, H.; Blug, A.; Carl, D.; Abt, F.: Omnidirectional Algorithm for the Full Penetration Hole Extraction in Laser Welding Processes, in Proceedings of the European Conference on Circuit Theory and Design ECCTD 2009, Antalya, Turkey (to be published).

[13]Company Anafocus, Avd. Isaac Newton s/n, Pabellón de Italia, Ático Parque Tecnológico Isla de la Cartuja, 41092 Sevilla, Spain.

[14]P. Dudek, P.J. Hicks, "A general-purpose processorper-pixel analog SIMD vision chip", IEEE Transactions on Circuits and Systems I: Regular Papers, Volume 52, Issue 1, pp. 13 - 20, Jan. 2005.

[15]A. Rodriguez-Vazquez, G. Linan-Cembrano, L. Carranza, E. Roca-Moreno, R. Carmona-Galan, F. Jimenez-Garrido, R. Dominguez-Castro, S.E. Meana, "ACE16k: the third generation of mixed-signal SIMDCNN ACE chips toward VSoCs", IEEE Transactions on Circuits and Systems I: Regular Papers, Volume 51, Issue 5, pp. 851 - 863, May 2004.

[16] Abt, F.; Blug, A.; Nicolisi, L..; Dausinger, F.; Weber, R.,; Tetzlaff, R.; Carl, D.; Höfler, H.: CLOSED LOOP CONTROL OF LASER WELDING PROCESSES USING CELLULAR NEURAL NETWORK CAMERAS - EXPERIMENTAL RESULTS, Proc. of the 28th In-ternational Congress on Applications of Lasers \& Electro-Optics (ICALEO 2009), 2009, Orlando, FL, USA, p1582-1591.

[17] Abt, F.; Blug, A.; Nicolosi, L.; Weber, R.; Tetzlaff, R.; Carl, D.; Höfler, H.: Laserschweißprozesse durch schnelle, pixelparallele Bildverarbeitung mit Zellularen Neuronalen Netzwerken (CNN) regeln, European Automotive Laser Applications (EALA) 2010, Bad Nauheim, Germany, p177-188.

(Received: July 08, 2009, Accepted: June 28, 2011) 